\title{
L'évolution du contrat en droit québécois : instrument d'équité entre partenaires sociaux, entre organisations? À quelles conditions?
}

\author{
Marc-André Morency et Jeanne Simard ${ }^{1}$ \\ Université du Québec à Chicoutimi
}

\begin{abstract}
Tout n'est pas contractuel dans le contrat
\end{abstract}
Émile Durkeim

L'activité contractuelle fait partie de la vie quotidienne de tout individu et, naturellement, de tout gestionnaire. Plusieurs variantes peuvent se présenter à eux : du contrat simple, comme l'achat d'articles de papeterie pour l'organisation, aux rapports contractuels plus complexes sur le plan national et international, comme le franchisage, les contrats de sous-traitance, les ententes d'impartition, etc. Dans le monde actuel, les contrats encadrent la production et la circulation des richesses, et transcrivent de ce fait des besoins humains et sociaux de plus en plus variés. À tel point que pour certains, le contrat serait la forme la plus achevée du lien social. On en a fait une espèce d'idole constitutive du fait social, dictant désormais l'évolution des rapports à tous les échelons de l'activité humaine. À la limite, la conscience collective, sous une forme trompeuse, finit par se représenter le lien social comme un agrégat d'individus égoïstes se rencontrant sporadiquement pour conclure des ententes donnant force de loi suprême au calcul d'intérêts particuliers parfaitement rationalisés. Selon cette logique, nos relations avec les autres se limiteraient aux fins immédiates ou plus lointaines recherchées.

Mais dans la vie sociale, est-ce que les liens entre les individus se résument à des contrats, c'est-à-dire à des normes prédéterminées devant être respectées sous peine de sanction? Que deviennent alors les phénomènes moraux

\section{Est-ce que tout y est contractuel?}

de solidarité, de fraternité, les obligations non juridiques pourtant omniprésentes dans toute l'histoire de l'humanité? Durkheim nous invite à nous poser la question : lorsqu'on rédige ou signe un contrat, est-ce que tout $\mathrm{y}$ est contractuel? Ne devons-nous pas toujours y voir la place qu'ouvrent la réflexion, le dialogue, la délibération éthique, entendue comme pratique visant à dresser une hiérarchie de valeurs pivots et donc, de normes? Le contrat ne nous invite-t-il pas forcément à nous référer comme il se doit au cadre relationnel qui lui préexiste, qui l'encadre et qui subsiste après sa conclusion?

L'observation empirique montre pourtant sans difficulté que la pratique contemporaine du contrat est assurément plus complexe qu'il n'y paraît de prime abord. Cette pratique engage souvent à long terme un ensemble d'acteurs socio-économiques, et ce, au sein de formations sociales influant grandement sur les comportements. Pensons à la famille, au monde des affaires, aux ordres professionnels, aux nations autochtones, etc. Dès lors qu'ils se vouent de bonne foi à la recherche de l'équité, à la réalisation d'objets ou d'intérêts particuliers, les acteurs doivent le plus souvent aller au-delà des obligations définies par la loi et maîtriser une constellation de faits normatifs ou éthiques que le législateur ne peut entièrement prédéfinir ou dont le juge ne 
peut se saisir en lieu et place des parties au débat, advenant un conflit. En guise d'exemple, songeons aux quelque 600 négociations au Canada engendrées par les décisions de la Cour suprême, invitant les Autochtones et les autorités, ainsi que les communautés respectives, à négocier de bonne foi pour le long terme, les arrangements de la vie commune.

Prise au sens large, la création normative des parties prenantes trouve dans le travail contractuel une occasion privilégiée de mettre en œuvre une «juridicité» non étatique, avec les développements et les dérives alors possibles. On peut substituer au contrat simple, en principe clos, le contrat à long terme prévoyant des ajustements périodiques. Ces espaces relationnels constituent des mondes particuliers, des ordres sociaux complexes à comprendre et à maîtriser pour les citoyens, les gestionnaires, les organisations, les institutions.

Le propos de cet article vise à rappeler : 1) la nature et la fonction du contrat, mais plus particulièrement la construction volontariste qui s'y trouve exprimée; 2) l'étendue des interventions de l'État et des tribunaux dans la liberté contractuelle visant à contrer les injustices pouvant s'y produire; 3) la place que reconnaît désormais le Code civil du Québec aux nouvelles pratiques contractuelles qui structurent l'adaptation et la collaboration mutuelle entre les parties.

Ces développements nous permettront d'illustrer le fait que les nouveaux rapports contractuels, dans leur diversité et leur flexibilité, obligent désormais le citoyen et le gestionnaire à élargir leurs compétences traditionnelles et à développer leur réflexion éthique. Cette réflexivité accrue devra prendre en compte de nombreux phénomènes d'inter normativité et les conséquences prévisibles de l'exécution du contrat sur autrui, sur l'environnement ainsi que sur l'ensemble de la société. Elle devra refléter le fait que l'équité, en droit, n'est pas une notion entièrement déterminée, qu'elle est un fait social toujours à reconstruire dans un contexte donné.

\section{La construction volontariste qu'exprime le contrat}

L'existence de patrimoines privés et la capacité de les aliéner sont à la fois les conditions et les objets de la réalité contractuelle. Selon la conception classique, toujours dominante dans la doctrine juridique actuelle, le contrat se définit comme un accord de volontés, comme une entente entre au moins deux personnes (morales ou physiques) visant un objet existant ou à produire, un service à rendre. Pris ensemble, ces accords multiples construisent un ordre social, un ordre juridique privé, lesquels organisent et contrôlent les comportements des parties contractantes. Cet ordre juridique privé repose sur le respect de la parole donnée, principe essentiellement social, fondé sur l'honneur, mais parfois revêtu de considérations religieuses. Pour le canoniste médiéval, par exemple, un chrétien non fidèle à sa parole irait à l'encontre de la Vérité; il tromperait son prochain et commettrait alors un péché mortel! (Supiot, 2005) ${ }^{2}$

Affranchi au XIX $^{\mathrm{e}}$ siècle des entraves religieuses et étatiques des anciennes structures (celles de l'Ancien Régime), le contrat en vient à représenter la logique d'émancipation des volontés véhiculée par la Réforme et le Siècle des Lumières, logique qui tend à faire du contrat l'expression idéalisée de la liberté et du libre arbitre. En France, le Code Napoléon, adopté en 1804, 
reconnaît le principe de l'égalité de tous les citoyens; l'autonomie de leur volonté et leur capacité juridique de contracter sont alors enfin reconnues. La bourgeoisie commerçante, industrielle, financière a pris la place des anciens détenteurs du pouvoir.

\section{Les règles qui régissent les contrats, de nature essentiellement instrumentale, font très peu référence à une quelconque éthique.}

De ce côté-ci de l'océan, c'est également dans l'esprit du libéralisme que s'accomplit, en 1866, la codification du droit civil du BasCanada (Québec), largement inspirée par le Code Napoléon. Plus particulièrement, le droit des contrats ne prévoit alors, presque exclusivement, que des règles supplétives de volonté, «qui peuvent être outrepassées par les parties qui désirent organiser autrement leurs relations » (Beaudoin, Jobin : 2005) ${ }^{3}$. Les règles qui régissent les contrats, de nature essentiellement instrumentale, font très peu référence à une quelconque éthique, que ce soit vis-à-vis la collectivité ou les individus. Louise Rolland précise :

Cette doctrine du «laissez-faire et laissezpasser » permettait à l'individualisme d'absorber la justice et la solidarité sociale. Un individu libre sauvegarde forcément ses intérêts. Par conséquent, un contrat auquel on a volontairement consenti est forcément juste, ce qui écarte toute la valeur corrective d'un quelconque droit objectif (Rolland, $1999)^{4}$.

Toutefois, les analystes de ces révolutions qui ont cours depuis 250 ans ont mis en évidence que la liberté contractuelle n'est pas toujours source de justice et que les volontés des parties ne sont pas aussi autonomes ou aussi bien informées qu'on le suppose dans la théorie économique classique. Les représentations particulières ne peuvent échapper aux vices de forme, aux préjugés, aux inégalités affectant les contractants. C'est le cas certainement pour cette masse de justiciables qui ne possède pas les habiletés requises pour tirer avantage du contrat comme mécanisme privé de régulation ou qui n'a pas les moyens de s'offrir les services d'un notaire ou d'un avocat. Belley s'adressant aux notaires souligne que :

Les contrats, loin d'être une image d'harmonie sociale, représentent le plus souvent un «rapport de forces économiques », le "siège d'une lutte d'intérêts », un « rapport de conflit », un « champ de tensions économiques » à cause des inégalités entre les parties contractantes (Rolland, 1999) ${ }^{4}$.

Goldstein et Mestiri portent eux aussi un regard critique sur cette philosophie politique impliquant ou présupposant la liberté naturelle des contractants et leur égalité :

[...] l'égalité naturelle et la pensée raisonnable innée sont des mythes. La réalité se traduit par la loi du plus fort ou du plus malin. À côté des inégalités naturelles, les inégalités sociales se sont multipliées à l'époque moderne: inégalité entre employeur et salarié; inégalité entre entreprises mêmes entraînant l'existence d'entreprises dépendantes d'autres, comme en matière de sous-traitance, de concession commerciale, de franchise; inégalité entre professionnel et simple profane ou consommateur, etc. (Goldstein, Mestiri, $2003)^{5}$.

Il ne faut pas se le cacher; si tous les individus qui contractent avaient un niveau de développement éthique élevé, c'est-à-dire si la solidarité et l'équité étaient intériorisées, de telles inégalités n'existeraient pas. Or dans les faits, ce niveau de développement est loin d'être atteint par l'ensemble des partenaires. Le psychosociologue et philosophe américain Lawrence Kohlberg (1969) ${ }^{6}$ avait ce problème en vue au moment d'élaborer sa 
théorie des trois niveaux de développement de la conscience chez l'individu. En voici les principaux aspects :

- Au premier niveau, l'individu prend ses décisions en fonction de son intérêt personnel. Si l'individu obéit aux normes, c'est par crainte de punitions. Il agit pour obtenir des récompenses ou par anticipation d'une réciprocité (le donnant-donnant).

Les récents scandales financiers nous ont donné maints exemples de gestionnaires à travers le monde se situant à ce premier niveau, négligeant entièrement l'intérêt des autres parties, ou de la communauté ambiante, avec comme résultat les désastres que cela a $\mathrm{pu}$ occasionner sur le plan social, et en particulier pour les organisations qui toléraient ou encourageaient ces comportements (Anderson, Enron, Nortel, Norbourg, etc.). On comprend qu'à ce niveau, les individus peuvent très bien se situer en marge de la légalité, en espérant échapper aux rigueurs de la justice, en l'occurrence, aux rigueurs du droit pénal. Aristote définit ce comportement comme relevant d'une nécessité, d'un état de nature. L'individu obéit, sans réfléchir, à ses pulsions de prédateur.

\section{Au deuxième niveau, l'individu est plus conscient; il prend en compte le contexte social auquel il appartient.}

- Au deuxième niveau, l'individu est plus conscient; il prend en compte le contexte social auquel il appartient. Cependant, cette conscience se limite à des groupes particuliers comme sa famille, ses pairs, son pays, etc.

Comme le renforcement est à ce niveau crucial, l'individu adopte un comportement qui correspond aux structures de renforcement perçues. Certains individus peuvent élargir leur perspective analytique pour y incorporer l'ensemble de la société dont ils font partie. Ils respectent grosso modo les lois, les règlements et les règles de déontologie, mais un peu comme des automates, sans remettre en question le fondement éthique (les valeurs pivots) de ces normes. Ils raisonnent en quelque sorte de la façon suivante lorsque vient le temps de prendre une décision : «s'il n'est pas interdit par des règles précises explicites, alors mon comportement est permis. » Le philosophe André Comte-Sponville écrit qu'on peut alors à la limite être en présence d'un salaud légaliste : «Aucune loi n'interdit l'égoïsme. De quel droit me reprochez-vous d'être égoïste? Je paie mes impôts, je n'ai jamais tué ni volé, je m'arrête aux feux rouges... Je ne vais pas, en plus, me faire du souci pour les pauvres! » (Comte-Sponville, 2004) ${ }^{7}$.

- Au troisième niveau, l'individu va au-delà des attentes des tiers pour déterminer ses décisions. S'il respecte la volonté du législateur, il est tout de même conscient que les valeurs peuvent varier d'un individu à un autre et ainsi requérir de définir l'ordonnancement des valeurs à mettre en œuvre. À un niveau supérieur encore, auquel parviennent certains rares individus, la personne embrasse une perspective universelle qui dépasse sa communauté pour inclure le monde entier, les générations futures, la nature et même des dimensions spirituelles.

Les individus orientent leurs actions en fonction des principes de justice, d'équité et de respect des droits, par exemple, et ce, peu importe ce qu'en pensent les autres. Leur prise de décision peut même aller jusqu'à la désobéissance civile. Ils acceptent les conséquences de leurs choix. Le sociologue Luc Boltanski, l'éthicien Thierry Pauchand, le psychologue Stanislas Grof font en ce cas référence à des leaders dont le niveau spirituel dépasse la moyenne, comme chez Gandhi, 
l'actuel dalaï-lama, Vaclav Havel, Mandela, etc.

La conception de la «justice» contractuelle sera différente selon le niveau de développement de la conscience de l'individu. Ceux du premier niveau font tous les contrats qu'ils veulent et ce, peu importe les conditions de violence qu'ils impliquent. Une seule valeur prime : il faut absolument en tirer avantage et ne pas se faire prendre si cela engendre des iniquités ou repose sur l'illégalité. Ceux du deuxième niveau, dont ferait partie la majorité des adultes, n'ont peut-être pas la maîtrise des valeurs éthiques, mais ils se conforment aux règles obligatoires édictées par le législateur en matière d'équité contractuelle. Enfin, les individus du niveau trois, qui selon certaines études formeraient environ $20 \%$ de la population, ont conscience que les contrats s'insèrent dans un cadre relationnel, une réalité durable en émergence, affectant l'ensemble de la société. Ils agissent en opérant des choix sur les moyens et les fins, de façon à rencontrer et dépasser les circonstances de leur action.

\section{L'étendue des interventions de l'État et des tribunaux dans la liberté contractuelle}

Devant les abus évidents engendrés par la liberté contractuelle et le faible développement de la conscience atteint par les individus, l'ordre juridique étatique (l'État) s'est vu pressé de réaffirmer les vertus de certains principes de justice. Ces interventions autoritaires, auxquelles aucun contractant ne peut déroger, paraissaient nécessaires au maintien de l'organisation sociale ainsi qu'à la création de rapports plus durables et équitables entre cocontractants à l'échelle supranationale.

\section{L'État développe ses interventions selon deux axes.}

L'État développe ses interventions selon deux axes. D'une part, il énonce des dispositions d'ordre public de direction visant la protection de l'intérêt général de la société ou la lutte contre les injustices systémiques. Au plan économique, «le législateur a édicté des règles visant à protéger la libre concurrence ainsi que les limitations aux prix et aux salaires afin de contrer l'inflation » $\left(\right.$ Karim, 1999) ${ }^{8}$. Sur le plan humain, il a tenu à prohiber expressément certains contrats ayant pour objet le vol, le meurtre, le trafic de stupéfiants, le trafic d'organes, les contrats de prêt destinés à des activités criminelles. D'autre part, le législateur énonce des dispositions d'ordre public de protection visant à rétablir l'équilibre des intérêts particuliers. Le législateur accorde une protection particulière aux individus ou aux groupes sociaux les plus vulnérables, soient les locataires résidentiels, les emprunteurs, les consommateurs, les adhérents, les salariés, les mineurs et les majeurs protégés ${ }^{1}$. Parmi ces protections, on trouve la possibilité ultime de faire dissoudre rétroactivement un contrat et de replacer les parties dans la position juridique et économique où elles se trouvaient avant la conclusion de l'engagement annulé, en vertu des principes que Durkheim reconnaissait au droit « restitutif ». On a aussi prévu la diminution des effets d'une clause, la réduction des obligations d'une partie, la condamnation à des dommages-intérêts.

Reconnaissant pour sa part l'évolution des mœurs et des usages, le législateur a voulu protéger, mais plus discrètement, le libre exercice de la liberté contractuelle et, donc, de promouvoir la justice et l'équilibre en codifiant plus spécifiquement certains instruments conceptuels flous à fondements moraux et donc coutumiers, comme la «bonne foi», «l'équité », «l'abus de droit» et la 
«raisonnabilité»; ces concepts viennent alors encadrer la formation, l'exécution et l'extinction de tous les contrats. L'utilisation d'une notion floue dans une loi a pour conséquence que le contenu n'est pas spécifié définitivement par le législateur; ce sont plutôt les tribunaux, les arbitres et les parties au contrat qui devront en quelque sorte les interpréter et en fixer les termes au fur et à mesure de l'évolution des pratiques et du contexte. Le législateur suppose en quelque sorte qu'existent des processus de délibération éthique dans la vie courante.

Voici quelques exemples de ces dispositions générales, dans le Code civil du Québec, qui affectent les obligations et les relations contractuelles dans cet esprit ${ }^{9}$ :

6. Toute personne est tenue d'exercer ses droits civils selon les exigences de la bonne foi.

7. Aucun droit ne peut être exercé en vue de nuire à autrui ou d'une manière excessive et déraisonnable, allant ainsi à l'encontre des exigences de la bonne foi.

1375. La bonne foi doit gouverner la conduite des parties, tant au moment de la naissance de l'obligation qu'à celui de son exécution ou de son extinction.

1434. Le contrat valablement formé oblige ceux qui l'ont conclu non seulement pour ce qu'ils y ont exprimé, mais aussi pour tout ce qui en découle d'après sa nature et suivant les usages, l'équité ou la loi.

Mais qu'est que l'on entend par l'expression «bonne foi »? Dans ses commentaires, le Ministère de la Justice écrivait en 1994:

Équivalent juridique de la bonne volonté morale et intimement liée à l'application de l'équité, la bonne foi est une notion qui sert à relier les principes juridiques aux notions fondamentales de justice. Sa codification, dans le domaine des obligations, devrait contribuer à inspirer tous les actes juridiques, principalement les contrats, dans leur conclusion, leur exécution et leur extinction, certes, mais aussi a fortiori, dans leur interprétation.
Le philosophe André Comte-Sponville nous donne un aperçu de la complexité de la bonne foi et de ses limites :

La bonne foi est une sincérité à la fois transitive et réflexive. Elle règle, ou elle devrait régler, nos rapports à autrui aussi bien qu'à nous-mêmes. Elle veut, entre les hommes comme à l'intérieur de chacun d'entre eux, le maximum de vérité possible, d'authenticité possible, et le minimum, en conséquence, de truquages ou de dissimulations. Il n'y a pas de sincérité absolue, mais non plus d'amour ou de justice absolue: cela n'interdit pas d'y tendre, de s'y efforcer, de s'en approcher parfois quelque peu... La bonne foi est cet effort, et cet effort est déjà une vertu (Comte-Sponville, 1995$)^{7} \quad$ (Les italiques sont de nous).

Cette conception de la bonne foi signifie qu'il n'y a pas de place dans le contrat pour le subterfuge. Concrètement, les tribunaux ont, à plusieurs occasions, sanctionné des contrats où l'un des cocontractants n'avait pas divulgué toute l'information qu'il détenait par rapport à l'objet de l'obligation. Ils ont aussi obligé une certaine constance dans le comportement des cocontractants. «Ainsi, une partie à un contrat n'est pas autorisée à invoquer subitement la lettre d'un contrat et demander la résiliation de ce dernier lorsqu'elle a approuvé les agissements de son débiteur sans protester pendant des années » (Lefebvre, 2000) ${ }^{10}$.

Un large courant de pensée contemporain, tant en Europe, qu'en Amérique, ajoute que la bonne foi ne devrait pas seulement comprendre la confiance, l'honnêteté et la probité, de la formation jusqu'à l'extinction des contrats, mais aussi la fraternité, la solidarité et la loyauté, c'est-à-dire que doit prévaloir un esprit de collaboration à long terme entre les parties. Le professeur français François Terré résume cette position de la façon suivante : 
«Loyauté, solidarité, fraternité », telle serait la «nouvelle devise contractuelle». En vertu de celle-ci, on assisterait au "dépassement d'une conception [...] individualiste et antagoniste du contrat où chacun veillait à la défense de ses propres intérêts ». Ce serait à chaque contractant de prendre en considération les intérêts de son partenaire, et même, pourquoi pas, de les privilégier. Quoi de plus naturel, au demeurant, puisque la référence à la bonne foi commanderait d'aimer (son contractant) comme un frère, cette aspiration fraternelle se déployant dans le droit des contrats en un souci d'altérité et de générosité apte à rendre l'humain vraiment humain (Terré, Simler, Lequette, 2002) $)^{11}$.

Mais cet idéal de justice contractuelle, qui implique des acteurs travaillant au troisième niveau de développement de la conscience défini par Kohlberg, celui de la création éthique, jusqu'où peuvent aller le législateur et les tribunaux pour l'encadrer? Doivent-ils se contenter d'en promouvoir l'émergence? Ou bien doivent-ils s'impliquer et tenter de définir ce développement social? Les avis sont à cet égard très partagés. Au Québec, cette question a été souvent abordée ces dernières années.

\section{Jusqu'où le législateur et les tribunaux doivent-ils encadrer la complexité des rapports contractuels?}

Sans aller jusqu'à exiger que le contrat devienne un haut lieu de sociabilité et d'amitié, certains groupes sociaux ont exercé des pressions considérables, à l'occasion de la réforme du Code civil du Québec, en 1994, afin que les codificateurs reconnaissent pour tous les justiciables (comme l'on fait l'Allemagne, l'Italie et la Suisse) deux nouveaux motifs d'ordre public d'annulation ou de révision d'une entente par un tribunal; l'imprévision et la lésion étendue à tous les majeurs. L'imprévision aurait légitimé l'intervention judiciaire dès qu'il y aurait eu un déséquilibre au cours de l'exécution d'un contrat en raison d'événements extérieurs non conformes aux attentes initialement exprimées (perte d'une source importante de revenus pour un des contractants, etc.). En outre, en cas de lésion entre majeurs, l'intervention judiciaire aurait permis de prendre en compte un déséquilibre présent lors de la formation d'un contrat en raison de l'exploitation d'une partie par l'autre ${ }^{12}$.

Or, ces importants pouvoirs de correction ne furent pas retenus dans la révision du Code civil, au grand dam de certains qui y ont vu la victoire de l'idéologie du capitalisme de l'accumulation primitive, de la déréglementation et de la privatisation. Au nom de la sécurité dans les conventions et de la préservation du principe de l'autonomie de la volonté, le législateur a plutôt considéré que les parties doivent demeurer libres d'inclure ou non de telles clauses dans leurs ententes. À titre d'exemple, les contrats commerciaux de longue durée peuvent incorporer « des clauses d'indexation ou des clauses de révision, de sauvegarde ou de hardship, qui permettent de modifier les prix ou de réaménager le contrat, dans l'hypothèse ou des changements dans la conjoncture viendraient à le déséquilibrer gravement » (Pineau, Burman et Gaudet; 2001: 285) ${ }^{13}$. D'autre part, une étude empirique auprès de plusieurs praticiens en droit de la famille (avocats, notaires, mais aussi des thérapeutes conjugaux) a mis en évidence que $40 \%$ des contrats de mariage incorporent déjà des mécanismes de mise à jour périodique de leurs termes à la lumière de circonstances nouvelles (Roy; 2006: 690) $)^{14}$.

Ce refus du législateur d'encadrer encore plus la complexité du monde actuel et de gérer 
toutes les situations conflictuelles est pourtant bien compréhensible. La tâche est beaucoup trop ardue, et certainement impossible à réaliser. Plus judicieusement encore, il faut même considérer que l'État, dans ses opérations habituelles, ainsi que les tribunaux de droit commun, n'ont tout simplement pas leur place dans la chambre à coucher de la délibération éthique! En effet, il faut considérer que d'autres normativités que le droit, dans la relation contractuelle, entre autres, peuvent être mobilisées pour la réalisation d'un projet commun. Les normes morales, culturelles, scientifiques, médicales, religieuses sont de fait toujours mises à contribution, s'articulant les unes aux autres, lorsqu'il s'agit de construire une solution humaine aux conflits.

C'est ainsi que, dans un contexte évolutif, le contrat, selon le professeur Jean-Guy Belley $^{15}$,

[... pourrait être appelé [...] à exercer une fonction névralgique dans l'adaptation des systèmes juridiques aux défis de régulation posés par la complexité des sociétés parvenues au stade de la modernité avancée et de la globalisation des échanges. Dans un régime reposant de plus en plus sur une production décentralisée et pluraliste des normes d'action, le contrat deviendrait un vecteur fondamental de communication et d'ajustement entre les sphères sociales spécialisées, en particulier celles du droit et de l'économie (Belley; $1999: 5)^{15}$.

C'est ainsi qu'on remarque, depuis quelques années, un net recul de l'utilisation des tribunaux de droit commun dans le règlement des conflits. L'arbitrage privé, la médiation se judiciaire de la puissance publique. L'État, tiers garant des lois, fait place à la justice convenue entre les parties, lesquelles réfèrent ensemble de leur désaccord devant un tiersarbitre de leur choix, qui voit à préserver la relation contractuelle ainsi que la confidentialité essentielle en affaires. Mais cette approche permet aussi aux parties de construire leurs solutions en faisant intervenir d'autres normes sociales, culturelles et religieuses qui auraient été jugées non pertinentes lors d'un procès. La juge Louise Otis, de la Cour d'appel du Québec, qui est une figure dominante dans la diffusion des médiations extrajudiciaires et dans la modernisation des tribunaux administratifs (même à l'ONU), a bien exprimé le tournant décisif de notre système de médiation au Québec dans une conférence donnée à l’Université de Montréal en 2004.

Je pense aussi à ce litige entre des commerçants québécois d'origine libanaise et chinoise qui avaient vécu l'essentiel de leur vie dans leur pays d'origine. C'est par l'énonciation de leurs valeurs culturelles qu'ils ont fini par dénouer l'impasse face à la résiliation d'un bail commercial. Le oui du commerçant libanais modulé par des conditions restées incomprises et le silence poli du commerçant chinois qui, lors de son procès, était respectueusement demeuré muet, ont finalement trouvé un chemin de rencontre. L'énonciation de leurs valeurs culturelles a pu se manifester en un lieu plus informel - quoique solennel et respecté - qui laissait libre cours à leur expression (Otis; $2004: 22)^{16}$. 
L'utilisation de cette «nouvelle offre de justice» au Québec (désormais connue et enseignée dans plusieurs pays et continents du monde) montre que de nombreux acteurs se reconnaissent de plus en plus l'obligation de structurer progressivement la hiérarchie des normes et des valeurs, bref à produire l'éthique dans la variété des contextes dans lesquels se réalisent les échanges (Rolland, $1999)^{4}$. On invite les acteurs à entrer dans un processus de communication et de négociation coopérative visant la vie à long terme de la collectivité, selon des principes mutualistes fondés essentiellement sur une éthique de la responsabilité. Ainsi, les acteurs sont conviés à produire et à renouveler le sentiment de justice et de fraternité, à l'imposer tôt ou tard dans les faits et dans les structures. Idéalement, c'est toute la collectivité qui, par planification stratégique, par exemple, ou autrement, fera l'éthique dominante.

Dans un cadre élargi à la réalité mondiale, la prudence commande de reconnaître que même si des individus ont atteint des niveaux élevés de conscience, il demeure inévitable que certains facteurs internes dans l'organisation à laquelle ils appartiennent, comme le style de gestion de la haute direction, les obligent parfois à opérer à un niveau de développement inférieur. Selon Diane Girard, consultante en éthique auprès d'organisations publiques et privées,

[...] même lorsque l'individu possède une capacité de raisonnement moral élevée, il n'a pas nécessairement une volonté suffisante ou le courage de faire ce qui lui semble être le mieux dans ces circonstances. Des facteurs propres au contexte organisationnel ainsi que les caractéristiques de son poste viennent aussi influencer sa décision de façon importante (Girard : 2004; 85) ${ }^{17}$.
Compte tenu de la prégnance des forces «automatiques» du marché global et de l'extrême réticence de certains États à créer à l'échelon mondial un ordre public démocratique et universel, le professeur JeanGuy Belley demeure réaliste, sinon sceptique, sur les véritables choix des parties contractantes. Les lois du marché global se faisant «métanormes», venant de ce fait chapeauter l'ensemble des ordres sociaux nationaux, des dérives éthiques peuvent de plus en plus être constatées. Encouragées par des institutions «internationales » comme l'OMC, l'OCDE, la Banque mondiale, le FMI, les grandes entreprises coupent de plus en plus leurs liens avec leur nation d'origine, la liberté de contracter devant opérer par-delà les frontières nationales. Elles exercent d'énormes pressions afin que les États-nations abolissent les réglementations faisant obstacle à la libre circulation des produits, des services et des capitaux. La «contractualisation » de la société, laquelle nourrit une philosophie " contractualiste », fait ainsi reculer les minces avancées du droit du travail et de la politique sociale, réalisées dans quelques pays, au siècle dernier. Selon Belley,

L'invocation des contraintes du marché mondial et des nouvelles exigences de l'économie globale diminue toutefois la pression que les considérations du contrat communautaire peuvent exercer sur les décisions des acheteurs et des gestionnaires de l'entreprise. Ces derniers sont invités à concilier aussi harmonieusement que possible les exigences du contrat communautaire avec celles du contrat normalisé. La responsabilité sociale de l'entreprise envers la communauté régionale reste subordonnée à l'impératif incontournable du profit et à la logique des allégeances multiples qu'une firme multinationale doit maintenir concurremment (Belley; $1998: 221)^{15}$. 
Tant que n'existeront pas un ordre public mondial et des mécanismes d'application efficace, il faudra s'attendre aux pires abus contractuels de la part des grands prédateurs du monde. On revient en effet, à l'échelon mondial, au premier niveau du développement personnel, à des rapports fondés sur la violence. Tout devient négociable par contrat, car tout devient marchandise, y compris la pensée, la création, les éléments de l'environnement, la santé, l'éducation, l'être humain et son corps (Chemillier-Gendreau : 2003). Plusieurs pays du tiers-monde en font largement les frais sur le plan économique et humain. En acceptant de se livrer au marché mondial, ils ont renoncé à sauvegarder leur société distincte. Pour plusieurs observateurs, la réalité de ces rapports complexes évoque la perspective d'une « reféodalisation » du monde si tant est que le capitalisme n'ait jamais été autre chose qu'un phénomène produit et marqué de façon irrévocable par ses origines féodales (Supiot : 2004; 166) $)^{2}$. La direction des grandes entreprises, par un maillage de réseaux de contrats occulte, s'assure de la dépendance à long terme des cocontractants. Des critères d'efficacité et de qualité leur sont imposés, sans qu'ils puissent vraiment réagir. L'imposition des normes ISO (qui sont des normes privées, élaborées par des agences présumées indépendantes) en est un bel exemple. Les révolutions bourgeoises du $\mathrm{XIX}^{\mathrm{e}}$ siècle n'auraient ainsi jamais été autre chose qu'un changement à la tête du monde féodal, et l'évolution du droit des contrats, au centre même de ces bouleversements, nous révélerait maintenant ce secret bien mal gardé, camouflé derrière la bannière de la liberté, de la fraternité, et de l'égalité...

Il nous apparaît urgent, sur le plan international, qu'une prise de conscience s'accomplisse et qu'un nouvel ordre public se constitue, sinon les belles avancées que le Québec a réalisées sur le plan interne dans ses politiques sociales, dans sa codification des lois et dans ses nouvelles procédures de médiation et d'administration de la justice, seraient mises en péril. Le professeur Gil Rémillard, ancien ministre de la Justice et à ce titre responsable de la réforme du Code civil du Québec, mentionnait lors d'une conférence en 2005 à l'Université de Montréal, que notre Code civil est maintenant traduit en plusieurs langues. Plusieurs pays comme la Roumanie, l'Argentine, la Bulgarie et même la Chine s'en seraient inspirés pour effectuer leurs propres réformes juridiques nationales, en évitant les difficultés d'adaptation qui sont le fait des longs textes de la common law tout empreinte des circonstances locales des litiges. Madame Louise Otis, juge à la Cour d'appel du Québec, vient également de travailler pendant de longs mois, avec une panoplie d'experts internationaux, à la modernisation des tribunaux administratifs de l'Organisation des Nations Unies, en y transposant le fruit des avancées québécoises en la matière. Ces avancées permettent de penser que si petit qu'il soit, le Québec saurait participer à cette évolution internationale jugée de plus en plus par les experts et les secteurs actifs de la société civile comme indispensable.

\section{Notes et références}

1 Marc-André Morency est professeur de sociologie au Département des sciences humaines à l'Université du Québec à Chicoutimi; Jeanne Simard est avocate et professeure de droit au Département des sciences économiques et administratives, à l'Université du Québec à Chicoutimi; les auteurs tiennent à remercier les professeurs Denis Bourque et Yves Hallée ainsi que Me Benoît Lapointe pour leurs judicieuses remarques.

2 Alain Supiot (2005), Homo juridicus. Essai sur la fonction anthropologique du Droit, Paris, Édition du Seuil, pp. 153-169. 
3 Jean-Louis Beaudouin, Pierre-Gabriel Jobin (2005), Les obligations, 5e éd., Les Éditions Yvon Blais, Cowansville.

4 Louise Rolland (1999), «Les figures contemporaines du contrat et le Code civil du Québec», Mc Gill Law Journal, vol. 44, pp. 910-913.

Louise Rolland (2006), «Les frontières de l'ordre contractuel : les traçages économiques et juridiques », Les Cahiers de droit, vol. 47, $\mathrm{n}^{\mathrm{o}} 1$, p. 13

Le texte est disponible sur le site internet suivant : http//www.journal.law.mcgill.ca/abs/vol44/4rolla.pdf

5 Gérald Goldstein et Najla Mestiri (2003), «La liberté contractuelle et ses limites - Étude à la lueur du droit civil québécois», dans B. Moore dir., Mélanges Jean Pineau, Montréal, Les Éd. Thémis, p. 314.

6 Lawrence Kohlberg (1969), Stage and sequence: The cognitive-developmental approach to socialization, dans D.A. Goslin (ed), Handbook of socialization theory and research, p. 347-380, Chicago : Rand McNally.

7 André Compte-Sponville (2004), Le capitalisme est-il moral ? Sur quelques ridicules et tyrannies de notre temps, Paris, Albin Michel, p. 104.

André Compte-Sponville (1995), Petit traité des grandes vertus, Paris, PUF/Perspectives Critiques.

8 Vincent Karim (1999), «L'ordre public en droit économique : contrats, concurrence, consommation», Les Cahiers de droit, vol. 40, 403-435.

9 En tout, 86 articles du Code civil du Québec évoquent les termes bonne foi ou mauvaise foi.

10 Brigitte Lefebvre (2000), «Liberté contractuelle et justice contractuelle : le rôle accru de la bonne foi comme norme de comportement», Développements récents en droit des contrats, $\mathrm{n}^{\circ} 129$, Cowansville, Les Éditions Yvon Blais, 40-70.

11 François Terré, Philippe Simler, Yves Lequette (2002), Droit civil. Les obligations, 8 édition, Paris, Dalloz.

12 La lésion entre majeurs est déjà permise pour quelques cas particuliers comme à l'article 2332 C.c.Q.

qui permet, entre autres, la réduction du coût d'un prêt d'argent ou pour certains contrats de consommation. Dans ce dernier cas, peu de décisions ont été rendues.

13 Jean Pineau, Danielle Burman, Serge Gaudet (2001), Théorie des obligations, $4^{e}$ éd., Montréal, Éditions Thémis.

14 Alain Roy (2007), «10 ${ }^{\mathrm{e}}$ conférence Albert-Mayrand. La charte de vie commune ou l'émergence d'une pratique réflexive du contrat conjugal», à paraître dans 41 Revue juridique Thémis, env. 20 pages.

15 Cherchant à cerner cette complexité normative, légale, coutumière, éthique, appelée actuellement à structurer notre horizon décisionnel, dans une recherche empirique sur les politiques d'achat d'Alcan auprès de fournisseurs du Saguenay-Lac-Saint-Jean, Jean-Guy Belley a pu démontrer que plusieurs contrats ont été formulés dans une large mesure en vertu de règles extérieures au droit étatique. Parmi les ordres normatifs convoqués à ce «festin de la contractualité», on trouve par exemple les règlements de régie interne de l'entreprise, les normes nationales et internationales de l'industrie, les us et coutumes de la communauté régionale, la morale.

Jean-Guy Belley (1998), Le contrat entre droit, économie et société, Cowansville, Les éditions Yvon Blais.

Jean-Guy Belley (1999), «Les incertitudes du contrat», dans Ejan Mackaay, dir., Les incertitudes du droit: Uncertainty and the Law, Montréal, Éditions Thémis, p. 5.

16 Louise Otis (2004), «La transformation de notre rapport au droit par la médiation judiciaire », Université de Montréal, $23 \mathrm{p}$.

17 Diane Girard (2004), «Les dimensions psychologiques de l'éthique : l'impact du contexte organisationnel », Interactions, vol. 8, $\mathrm{n}^{\mathrm{o}} 1,81-99$.

18 Monique Chemillier-Gendreau (2003), «Pour une éthique de l'économie. Le droit, élément de frein ou de progrès ? », Paris, Organisation des Nations Unies pour l'éducation, la science et la culture, 33 p. http://unesdoc.unesco.org/images/0013/001305/130503f.pdf 


\section{Pub}

\section{Maîtrise en gestion de projet}

